\title{
$\overline{\text { Potential Origins of a Quantitative Equivalence Between Gravity and Light }}$
}

\author{
Michael A. Persinger*
}

Laurentian University, Sudbury, Ontario, Canada

\begin{abstract}
Quantitative estimates indicate that the energy equivalence over the universe's length from gravitational forces between the rest mass of all photons each separated by Planck's length is a quantum of energy within the frequency band of visible light. The convergence of values between dynamic pressure within the universe and the density of photons within a cross-section of unit space with a thickness that reflects the visible wavelengths as well as the shared metric of $10^{104} \mathrm{~m}^{4} \mathrm{~kg}^{-2}$ for transformation of $\mathrm{G}$ to dynamic pressure and the number of photon equivalents within the universal mass $\left(10^{52} \mathrm{~kg}\right.$ divided by $10^{-52} \mathrm{~kg}$ per rest photon) support this inference. Both Planck's constant and the precession frequency of neutral hydrogen are potentially derivable from the intrinsic assumptions. These results suggest the relationship between gravity and electromagnetic quantum may be a quantitative solution rather than a qualitative derivation and may facilitate understanding of the particle-wave properties of light.
\end{abstract}

Keywords: Electromagnetism, Gravity, Light, Universe, Photon Mass.

\section{GRAVITATIONAL AND ELECTROMAGNETIC ENERGIES}

There have been multiple theoretical and qualitative approaches to understand the relationship between the phenomena defined as gravitation and electromagnetism, particularly light, integrated through micro- and macrospace [13]. Within a terrestrial framework Minakov et al. [4] described the conversion of a plane gravitational wave into electromagnetic radiation while Vladimirskii [5] measured enhancement in the order of $10^{-3}$ within $\mathrm{G}$ (the gravitational constant) with lower geomagnetic activity. Although there are both parsimonious and conceptual advantages for isolating equations that integrate gravitational and electromagnetic properties, there is the possibility that equivalences may be reflected more in quantitative relationships. In the present paper, evidence for the convergent validity for a numerical solution that employs the minima and maxima for mass, dynamic pressure, and energy is considered.

\section{A QUANTITATIVE SOLUTION}

According to Tu et al. [6] the upper limit for the rest mass of a photon may be $\sim 10^{-52} \mathrm{~kg}$. If we assume the minimal distance between two photons is Planck's length, a metric also suggested by some applications of Casimir interactions, then the gravitational force is between $10^{-45}$ and $10^{-44}$ $\mathrm{N}\left[\left(\sim 10^{-104} \mathrm{~kg}^{2}\right) / \sim 10^{-70} \mathrm{~m}^{2}\right] \cdot\left[6.67 \cdot 10^{-11} \mathrm{~m}^{3} \mathrm{~kg}^{-1} \mathrm{~s}^{-2}\right]$. If this intrinsic force from the smallest spatial increment is extended over the largest width of the visible universe of about $10^{26} \mathrm{~m}$ (assuming the universe is $\sim 13$ billions of years old), the energy peak energy would be within range of $10^{-19}$ to $10^{-18} \mathrm{~J}$.

*Address correspondence to this author at the Laurentian University, Sudbury, Ontario, Canada; Tel: 01-705-675-4824; Fax: 01-705-671-3844;

E-mail: mpersinger@laurentian.ca
This energy, when divided by Planck's constant is between $10^{14} \mathrm{~Hz}$ to $10^{15} \mathrm{~Hz}$ or within the order of magnitude of the band of the visible wavelength. For comparison Sivaram and Arun [7] showed that thermal gravitational radiation from compact stellar objects occurs within the $10^{16} \mathrm{~Hz}$ to $10^{21} \mathrm{~Hz}$ range. Given the uncertainty of the coefficients for the values of the rest mass of photons and the diameter of the visible universe, a more exact solution whose median value is within the visible spectrum ( 400 to $800 \mathrm{~nm}$ ) could be considered highly probable. If this solution is valid, then one might consider the electromagnetic energy associated with light to be an expression of the gravitational force between the rest mass of all photons spread over space and time as inferred by the width of the universe. One could also state that the energy equivalence over the universe's length from gravitational forces of all photon rest masses separated by Planck's length produces a gravitational energy that exhibits a frequency within the range of visible light.

\section{CONVERGENT VALIDITY}

The relationship between universal pressure and gravity has been entertained intermittently. Assuming a net density (2) of 1 proton mass $\mathrm{m}^{-3}$ Persinger [8] suggested that the resulting universal pressure $\left(\partial \mathrm{c}^{2}\right)$ would be $15 \times 10^{-11} \mathrm{~Pa}$. To obtain the equivalence in coefficient and units to converge with $\mathrm{G}$, an identity transform of $\mathrm{m}^{4} \mathrm{~kg}^{-2}$ was required between pressure and G. Assuming a width of the universe of $\sim 10^{26} \mathrm{~m}$, this resulted in a four dimensional metric of $10^{104}$ $\mathrm{m}^{4}$ and the required mass of $10^{52} \mathrm{~kg}$. This estimated mass for the universe is within an order of magnitude of other values derived from empirical estimates such as the estimated numbers of galaxies $\left(10^{11}\right)$ in the universe, numbers of stars $\left(10^{11}\right)$ within each galaxy and an average star mass of the sun $\left(10^{30} \mathrm{~kg}\right)$. It is relevant that the classic aggregate $8 \pi \mathrm{Gc}^{-2}$, which accommodates the second derivative of the surface of 
a sphere, is the value $1.9 \times 10^{-26} \mathrm{~m} \cdot \mathrm{kg}^{-1}$; a mass of $10^{52} \mathrm{~kg}$ results in a radius of $\sim 10^{26} \mathrm{~m}$.

With the estimated mass of the universe of $10^{52} \mathrm{~kg}$ and the upper limit of the estimated rest mass of the photon as $10^{-52} \mathrm{~kg}$, the total numbers of equivalents of rest-mass photons would be $10^{104}$. This value is an identity with the value of the four dimensional metric [8] required to satisfy the equivalence between $G$ and intrinsic pressure. Although various coefficients of metrics of the shape of space are obviously important for fine resolution of subatomic and theoretical particles $[9,10]$, the assumption here is that the functional volume of the universe is between $10^{77} \mathrm{~m}^{3}$ and $10^{78}$ $\mathrm{m}^{3}$. This means the average historical density of rest mast photons range from $10^{26}$ to $10^{27}$ photons $\mathrm{m}^{-3}$. The minimum numbers of photons within a thin slice defined by the range of wavelengths between $3 \cdot 10^{-7} \mathrm{~m}$ to $3 \cdot 10^{-6} \mathrm{~m}$ reflecting the derived frequencies $\left(10^{14} \mathrm{~Hz}\right.$ to $10^{15} \mathrm{~Hz}$ or) from the gravitational to electromagnetic "transformation" would be $\sim 10^{19}$ photons $\mathrm{m}^{-2}$.

To obtain the "force"-equivalent the rest mass of a photon moving at $\mathrm{c}$ was multiplied by the central range of frequencies of $10^{14} \mathrm{~Hz}$ and $10^{15} \mathrm{~Hz}$ (light) which would result in a value between $10^{-29}$ and $10^{-30} \mathrm{~N}$. With $10^{19}$ photons $\mathrm{m}^{-2}$ the net dynamic pressure from rest mass photons moving at $\mathrm{c}$ at the critical transformation energies between gravitational and electromagnetic forces would be between $10^{-11}$ and $10^{-10} \mathrm{~Pa}$. This is within the same range as the value [8] employed to estimate the mass of the universe.

A second source of convergence is apparent within the general field equation. The energy equivalence of the mass of the universe would be $\sim 10^{69} \mathrm{~J}$. From an estimated number of $10^{104}$ rest mass photons in the universe, this means that each photon would display an increment of about $10^{-35} \mathrm{~J}$. This value is within the order of magnitude of Planck's constant as well as the energy associated with one rotation of an electron's mass-energy equivalent around the Bohr magneton, assuming the fine structure velocity. However what is more relevant for the present theme is that $10^{-35} \mathrm{~J}$ over the age of the universe $\left(\sim 10^{17} \mathrm{~s}\right)$ is $10^{-18} \mathrm{~J}$ for increments of $1 \mathrm{~s}$. Consequently the energy band associated with the frequency of light would be the solution for the energy of the rest photon mass equivalent distributed over the age of the universe.

If the quantum of EM we perceive as light is the constant condition of the gravitational attraction between every photon at its rest mass spread across the universe, then there should be an intrinsic continuity reflected in a fundamental property. At the unit of velocity where $c^{2} \rightarrow c$, that is it approaches a value $=1$, the energy of the rest photon is $10^{-52} \mathrm{~J}$. When divided by Planck's time of $\sim 10^{-44} \mathrm{~s}$ the value is $10^{-8} \mathrm{~J}$ $\mathrm{s}^{-1}$ and for the time of one rotation of an electron (Bohr magneton), the average energy would be $10^{-8} \mathrm{~J} \mathrm{~s}^{-1} \cdot \sim 10^{-16} \mathrm{~s} \mathrm{rev}^{-1}$ $=\sim 10^{-24} \mathrm{~J}$. The frequency for this energy is $1.5 \mathrm{GHz}$ which is remarkably proximal, given the variability in measurements, to the precession frequency of neutral hydrogen atoms (1.42 $\mathrm{GHz}$ ), the most abundant substance in space. Although optimally the precise solution should be exactly equal to the precession frequency, such convergence would require more accurate identification of the magnitudes of the coefficients.

\section{A NECESSARY CAVEAT}

Based upon a Hubble's constant of $\sim 75 \mathrm{~km} \mathrm{~s}^{-1}$ per MParsec Persinger and Koren [11] quantified the expansion of various ranges of space occupied by matter. The Casimir effect was considered one possible means by which an expanding boundary could transmute virtual particles into real particles as quantum manifestations of vacuum energy [12]. Koren and Persinger [13] later calculated that to accommodate an intrinsic universal pressure of about $0.1 \mathrm{nPa}[8]$ the Casimir solution required a second boundary separated from the universal perimeter by $54 \mu \mathrm{m}$. The estimated power density $\left(0.45 \mathrm{~W} \mathrm{~m}^{-2}\right)$ associated with the blackbody electromagnetic emissions from this concentric ring around the universe's boundary would be within the mJy (1 Jansky $=10^{-26}$ $\mathrm{W} \mathrm{m}{ }^{-2} \mathrm{~Hz}^{-1}$ ) range at earth distances, which has been measured. The value is within the range measured in most distant galaxies with large reds shifts.

As indicated by Puthoff [2], gravity is an induced effect associated with changes in the quantum-fluctuation energy of the vacuum in the presence of matter. One of the consequences of vacuum quantum effects associated with an intrinsic frequency of zero point fluctuations is the creation of particles from the vacuum by external fields if the boundary conditions depend upon time. During this condition the transfer of external energy to virtual particles transforms them to measurable ones [12]. Because the universal boundary can be considered expanding the conditions for transforming virtual particles to matter might be satisfied. The presence of $0.45 \mathrm{~W} \mathrm{~m}^{-2}$ distributed over an estimated universal surface boundary of $4.5 \cdot 10^{53} \mathrm{~m}^{2}$ would result in the availability of $\sim 2 \cdot 10^{53} \mathrm{~J} \mathrm{~s}^{-1}$ or a mass equivalence of $10^{36} \mathrm{~kg}$ $\left(\sim\right.$ a million solar masses $\left.\mathrm{s}^{-1}\right)$. Although the estimated mass of the universe would be approximately matched in another $10^{16} \mathrm{~s}$, the proportion of change would be negligible during the life time of the human observer. However this minute change is present and could affect the assumptions employed in the present calculations.

\section{CONFLICT OF INTEREST}

None declared.

\section{ACKNOWLEDGEMENT}

None declared.

\section{REFERENCES}

[1] Hawking S. A brief history of time. Bantam: New York 1988

[2] Puthoff HE. Gravity as a zero-point-fluctuation potential. Phys Rev 1989; 39: 2333-42.

[3] Islam JN. Possible ultimate fate of the universe. Q J Royal Astron Soc 1977; 8: 3-8.

[4] Minakov AA, Nikolaenko AP, Rabinovich LM. Gravitational-toelectromagnetic wave conversion in electrostatic field of earthionosphere resonator. Radiofizika 1992; 35: 915-23.

[5] Vladmirskii BM. Measurements of gravitational constant and heliogeophysical electromagnetic perturbations. Biophysics 1995; 40: 915-23.

[6] Tu LC, Luo J, Gilles GT. The mass of the photon. Rep Prog Phys 2005; 68: 77-130.

[7] Sivaram C, Arun K. Thermal gravitational waves. Open Astron J 2011; 4: 65-71.

[8] Persinger MA. A simple estimate of the mass of the universe: dimensionless Parameter A and the construct of "pressure". J Phys Astrophys Phys Cosmol 2009; 3: 1-3. 
[9] Wu P, Yu H. Reconstructing the properties of dark energy from recent observations. J Cosmol Astropart Phys 2007; 10: 1-5.

[10] Bahcall NA, Ostriker JP, Pelmutter S, Steindhardt PJ. The cosmic triangle: revealing the state of the universe. Science 1999; 284 : 1481-8.

[11] Persinger MA, Koren SA. A theory of neurophysics and quantum neuroscience: implication for brain function and the limits of consciousness. Int J Neurosci 2007; 117: 157-75.
[12] Bordag M, Mohideen U, Mostepanenko VM. New developments in the Casimir effect. Phys Rep 2001; 353: 1-205.

[13] Koren SA, Persinger MA. The Casimir force along the universal boundary: quantitative solutions and implications. J Phys Astrophys Phys Cosmol 2010; 4: 1-5.

Received: April 19, 2012

Revised: May 18, 2012

Accepted: May 21, 2012

() Michael A. Persinger; Licensee Bentham Open.

This is an open access article licensed under the terms of the Creative Commons Attribution Non-Commercial License (http://creativecommons.org/licenses/by-nc/3.0/) which permits unrestricted, non-commercial use, distribution and reproduction in any medium, provided the work is properly cited. 